University of Nebraska - Lincoln

DigitalCommons@University of Nebraska - Lincoln

U.S. Environmental Protection Agency Papers

U.S. Environmental Protection Agency

2009

Combining Risks from Several Tumors Using Markov Chain Monte Carlo

Leonid Kopylev

U.S. Protection Agency

John Fox

U.S. Environmental Protection Agency

Chao Chen

U.S. Environmental Protection Agency

Follow this and additional works at: https://digitalcommons.unl.edu/usepapapers

Kopylev, Leonid; Fox, John; and Chen, Chao, "Combining Risks from Several Tumors Using Markov Chain Monte Carlo" (2009). U.S. Environmental Protection Agency Papers. 140.

https://digitalcommons.unl.edu/usepapapers/140

This Article is brought to you for free and open access by the U.S. Environmental Protection Agency at DigitalCommons@University of Nebraska - Lincoln. It has been accepted for inclusion in U.S. Environmental Protection Agency Papers by an authorized administrator of DigitalCommons@University of Nebraska - Lincoln. 


\title{
COMBINING RISKS FROM SEVERAL TUMORS USING MARKOV CHAIN MONTE CARLO
}

\author{
Leonid Kopylev, John Fox, and Chao Chen \\ National Center for Environmental Assessment US Environmental Protection Agency
}

\section{INTRODUCTION}

In animal bioassays, tumors are often observed at multiple sites. Unit risk estimates calculated on the basis of tumor incidence at only one of these sites may underestimate the carcinogenic potential of a chemical (NRC 1994). Furthermore the National Research Council (NRC, 1994) and Bogen (1990) concluded that an approach based on counts of animals with one or more tumors (counts of "tumor-bearing animals") would tend to underestimate overall risk when tumors occur independently across sites. On independence of tumors, NRC (1994) stated: “... a general assumption of statistical independence of tumor-type occurrences within animals is not likely to introduce substantial error in assessing carcinogenic potency." Also application of a single dose-response model to pooled tumor incidences (i.e., counts of tumorbearing animals) does not reflect possible differences in dose-response relationships across sites. Therefore the NRC (1994) and Bogen (1990) concluded that an approach that is based on well-established principles of probability

The views expressed in this article are those of the authors and do not necessarily reflect the views or policies of the US Environmental Protection Agency.

Uncertainty Modeling in Dose Response: Bench Testing Environmental Toxicity

Edited by Roger M. Cooke

Copyright (C) 2009 by John Wiley \& Sons, Inc. 
and statistics should be used to calculate composite risk for multiple tumors. Bogen (1990) also recommended a re-sampling approach, as it provides a distribution of the combined potency. Both NRC (1994) and Guidelines for Carcinogen Risk Assessment (US EPA 2005) recommend that a statistically appropriate upper bound on composite risk be estimated in order to gain some understanding of the uncertainty in the composite risk across multiple tumor sites.

This chapter presents a Markov chain Monte Carlo (MCMC) computational approach to calculating the dose associated with a specified composite risk and a lower confidence bound on this dose, after the tumor sites of interest (those believed to be biologically relevant) have been identified and suitable dose-response models (all employing the same dose metric) have been selected for each tumor site. These methods can also be used to calculate a composite risk for a specified dose and the associated upper bound on this risk. For uncertainty characterization, MCMC methods have the advantage of providing information about the full distribution of risk and/or benchmark dose. This distribution, in addition to its utility in generating a confidence bound, provides expected values of risks that are useful for economic analyses.

The methods presented here are specific to the multistage model with nonnegative coefficients fitted to tumor incidence counts (i.e., summary data rather than data on individual animals, as in the nectorine example; if data on individual animals are available, other approaches are possible), and they assume that tumors in an animal occur independently across sites. The nectorine example is used to illustrate proposed methodology and compare it with the current approach.

\section{COMBINING RISKS FOR THE MULTISTAGE MODEL}

The NRC (1994) has described an approach for combining risk estimates across tumor sites based on the multistage model: $P(d, \theta)=1-\exp \left[-\left(q_{0}+q_{1} d\right.\right.$ $\left.\left.+q_{2} d^{2}+\cdots+q_{k} d^{k}\right)\right]$, where $\theta=\left(q_{0}, q_{1}, \ldots\right), q_{i} \geq 0$, where $d \geq 0$ is the dose metric and $P(d, \theta)$ is the probability of response at dose $d$, with parameters $\theta$. For the multistage model and two tumor types, $A$ and $B$, assume $m>k$, and

$$
\begin{aligned}
& P_{A}\left(d, \beta_{A}\right)=1-\exp \left(-\left(\beta_{0 A}+\beta_{1 A} * d+\ldots+\beta_{k A} * d^{k}\right)\right), \\
& P_{B}\left(d, \beta_{B}\right)=1-\exp \left(-\left(\beta_{0 B}+\beta_{1 B} * d+\ldots+\beta_{m B} * d^{m}\right)\right) .
\end{aligned}
$$

Assuming independence of tumors

$$
P_{A \text { or } B}\left(d \mid \beta_{A}, \beta_{B}\right)=P_{A}\left(d, \beta_{A}\right)+P_{B}\left(d, \beta_{B}\right)-P_{A}\left(d, \beta_{A}\right) * P_{B}\left(d, \beta_{B}\right),
$$


we have,

$$
\begin{aligned}
P_{A \circ B}\left(d, \beta_{A}, \beta_{B}\right)= & 1-\exp \left(-\left[\left(\beta_{0 A}+\beta_{0 B}\right)+\left(\beta_{1 A}+\beta_{1 B}\right) * d+\ldots+\left(\beta_{k A}+\beta_{k B}\right) * d^{k}\right.\right. \\
& \left.\left.+\beta_{k+1 B} * d^{k+1}+\ldots+\beta_{m B} * d^{m}\right]\right) .
\end{aligned}
$$

Similarly, for more than two tumor types, the combined tumor model, $P_{c}$, is

$$
P_{c}(d, \theta)=1-\exp \left(-\left[Q_{0}+Q_{1} d+Q_{2} d^{2}+\ldots+Q_{k} d^{k}\right]\right)
$$

where $Q_{i}=\sum q_{i j}$, in which $i$ indexes the model "stages" 1 to $k$ and $j$ indexes the tumor sites, with $\theta=\left(Q_{0}, Q_{1}, \ldots, Q_{k}\right)$.

The benchmark dose method (Crump, 1984) consists of estimating a lower confidence limit for the dose associated with a specified increase in adverse response (i.e., increased risk) above the background level. Extra risk (ER) is a common choice:

$$
E R=\frac{P(d, \theta)-P(0, \theta)}{1-P(0, \theta)}
$$

The benchmark dose (BMD) for extra risk is the solution of the above equation when the left-hand side is fixed. Statistical inference for chemical risk assessment has mainly emphasized finding confidence limits for the BMD. For two tumors, extra risk is given by

$$
E R_{A \mathrm{or} B}\left(d, \beta_{A}, \beta_{B}\right)=\frac{P_{A \mathrm{or} B}\left(d, \beta_{A}, \beta_{B}\right)-P_{A \mathrm{or} B}\left(0, \beta_{A}, \beta_{B}\right)}{1-P_{A \text { or } B}\left(0, \beta_{A}, \beta_{B}\right)} .
$$

After simple algebra,

$$
\begin{aligned}
E R_{A \circ B}\left(d, \beta_{A}, \beta_{B}\right)= & 1-\exp \left(-\left[\left(\beta_{1 A}+\beta_{1 B}\right) * d+\ldots+\left(\beta_{k A}+\beta_{k B}\right) * d^{k}+\beta_{k+1 B} * d^{k+1}\right.\right. \\
& \left.\left.+\ldots+\beta_{m B} * d^{m}\right]\right) .
\end{aligned}
$$

BMD for combined risk $B M R_{A \text { or } B}$ is (after re-arranging and taking logarithms of both sides) the solution to the polynomial

$$
\begin{aligned}
-\log \left(1-B M R_{A \circ B}\right)= & \left(\beta_{1 A}+\beta_{1 B}\right) * d+\ldots+\left(\beta_{k A}+\beta_{k B}\right) * d^{k} \\
& +\beta_{k+1 B} * d^{k+1}+\ldots+\beta_{m B} * d^{m} .
\end{aligned}
$$

A similar polynomial equation applies when more than two tumors are observed in a bioassay. 


\section{BAYESIAN APPROACH}

A Bayesian approach for calculating a confidence bound on the BMD for composite risk can be implemented using WinBUGS (Spiegelhalter et al., 2003). This is a freely available software package that can be used to apply MCMC methods (e.g., Smith and Gelfand, 1992; Casella and George, 1992; Chib and Greenberg, 1995; Brooks, 1998; Gilks et al., 1998; Gelman et al., 2004). Gelfand et al. (1992) discusses MCMC methods involving constraints, as in the case of applying the multistage dose-response model to the nectorine data, where maximum likelihood estimates (MLE) for background coefficients for both tumors are on the boundary.

The use of MCMC methods (via WinBUGS) to derive a posterior distribution of BMDs for a single multistage model has been recently described by Kopylev et al. (2007). This methodology can be straightforwardly generalized to derive a posterior distribution of BMDs for combined tumor risk across sites, using the approach for composite risk described in the previous section.

The mode and 5th percentile of the resulting posterior distribution of the dose for a fixed extra risk provide estimates of the BMD and the BMDL ("lower bound") for composite tumor risk. Similarly, the mean and 95th percentile of the posterior distribution of the composite extra risk provide estimates of the expected extra risk and the upper bound on the extra risk.

\section{EXAMPLE}

The data for the nectorine bioassay are given in Table 5.1. Two tumor types were observed and no information beyond summary data is available.

A linear multistage model fits better-based on lowest values of the deviance information criterion (DIC) reported by WinBUGS-than a quadratic or cubic model for both adenoma and neuroblastoma tumors. Similarly a linear model is preferable for both tumors, based on minimizing AIC - based on BMDS (US EPA 2006) computations. The WinBugs results are obtained based on convergence of 3 chains with different initial values, and 50,000 burnin (i.e., the first 50,000 samples discarded) from 50,000 simulations each, using WinBUGS 1.4.1. The posterior distribution is obtained from the combined

\section{TABLE 5.1 Nectorine bioassay data}

\begin{tabular}{lcccr}
\hline & \multicolumn{4}{c}{ Concentration (ppm) } \\
\cline { 2 - 5 } Tumor Type & 0 & 10 & 30 & 60 \\
\hline Respiratory epithelial adenoma & $0 / 49$ & $6 / 49$ & $8 / 48$ & $15 / 48$ \\
Olfactory epithelial neoblastoma & $0 / 49$ & $0 / 49$ & $4 / 48$ & $3 / 48$ \\
\hline
\end{tabular}


150,000 samples from the three chains and thinned by retaining every 10th sample to reduce autocorrelation, resulting in 15,000 samples. A prior that is a mixture of a diffuse continuous distribution on a positive real line and a point mass at 0 was used for multistage parameters. In WinBUGS the prior was constructed by truncating a high-variance Gaussian prior distribution at -1 and using the "step" function to collect mass from the interval on the negative real line at 0 as a point mass. In a limited Monte Carlo simulation of the frequentist properties of the posterior, this was a reasonable choice for a range of scenarios we investigated.

The posterior distribution of the extra risk at dose $11 \mathrm{ppm}$ and $\mathrm{BMD}_{10}$ is shown on Figures 5.1 and $5.2(11 \mathrm{ppm}$ is chosen because it is close to the combined $\mathrm{BMD}_{10}$ ). The posterior distribution of the linear coefficient for the neoblastoma tumor is strongly bimodal with $40 \%$ of its mass at 0 and the rest of the mass continuously distributed. In a linear model, lack of the linear term implies no extra risk; that is, risk is the same at every dose. Since the extra risk for a linear model is a 1:1 function of the linear parameter, there is also bimodality of extra risk for neoblastoma in Figure 5.1. As the BMD in a linear model is inversely proportional to the linear term, for that $40 \%$ of neuroblastoma simulations in which the estimated linear term was zero, the BMD (Figure 5.2) could not be determined (mathematically it is infinite). In contrast, the linear parameter for the adenoma model has a unimodal posterior bounded away from zero (and so the extra risk distribution for adenoma

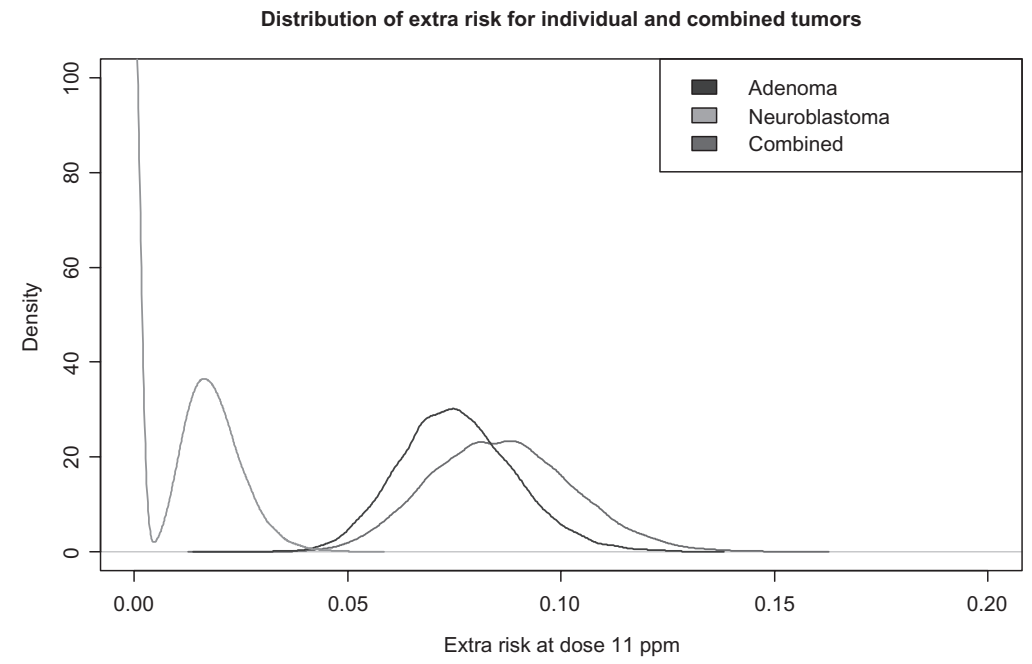

Figure 5.1 Distribution of the extra risk at dose $11 \mathrm{ppm}$ for individual and combined tumors for the nectorine example. Note that $40 \%$ of the distribution for neuroblastoma extra risk is at 0 , since the posterior of the linear parameter for this model has $40 \%$ of its mass at 0 . 


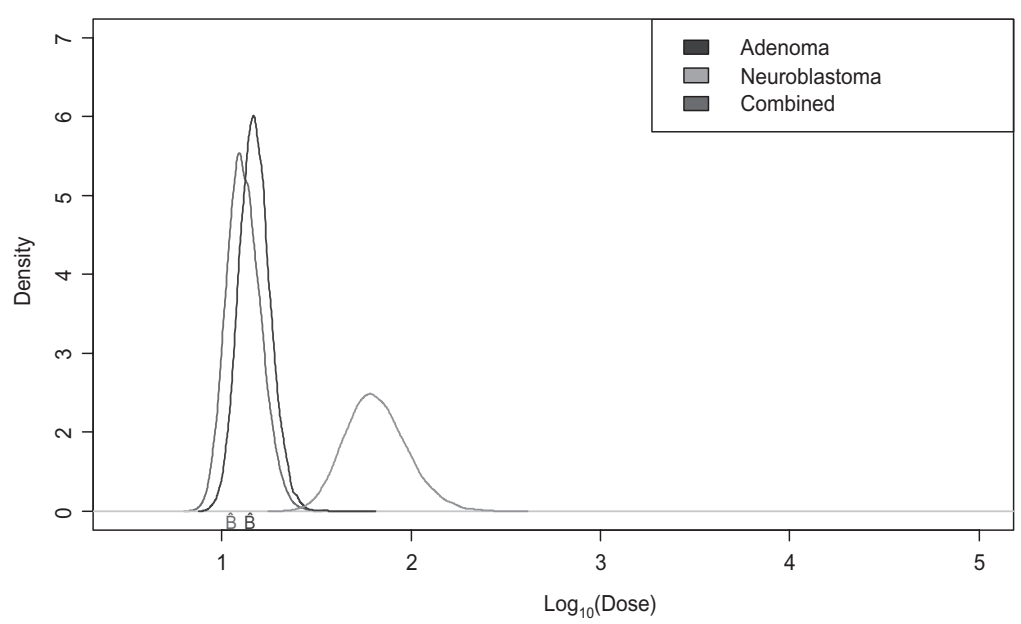

Figure 5.2 Distribution of the logarithm of $\mathrm{BMD}_{10}$ for individual and combined tumors in the nectorine example. Letters " $\mathrm{B}$ " on the $x$-axis indicate $\mathrm{BMD}_{10} \mathrm{~s}$ for adenoma and combined tumors. For $40 \%$ of neuroblastoma simulations (see Figure 5.1 caption), BMD cannot be determined (is infinite); thus the integral under the distribution for neuroblastoma is 0.6 .

TABLE 5.2 Statistics for individual and combined $\mathrm{BMD}_{10}$ for the nectorine example

\begin{tabular}{|c|c|c|c|c|c|c|}
\hline \multirow[b]{2}{*}{ Approach } & \multicolumn{3}{|c|}{ WinBUGS } & \multicolumn{3}{|c|}{ BMDS } \\
\hline & $\begin{array}{c}5 \text { th } \\
\text { Percentile } \\
\left(\mathrm{BMD}_{\mathrm{L}}\right)\end{array}$ & $\begin{array}{l}\text { Posterior } \\
\text { Mode } \\
\text { (BMD) }\end{array}$ & $\begin{array}{c}\text { 95th } \\
\text { Percentile } \\
\mathrm{BMD}_{\mathrm{U}}\end{array}$ & $\begin{array}{c}5 \text { th } \\
\text { Percentile } \\
\left(\mathrm{BMD}_{\mathrm{L}}\right)\end{array}$ & $\begin{array}{c}\text { MLE } \\
(\mathrm{BMD})\end{array}$ & $\begin{array}{c}\text { 95th } \\
\text { Percentile } \\
\text { BMD }_{U}\end{array}$ \\
\hline Adenoma & 11.06 & 14.20 & 20.63 & 11.32 & 15.17 & 22.79 \\
\hline Neuroblastoma & 40.63 & $\&$ & $\&$ & 39.91 & 70.11 & 153.60 \\
\hline Combined & 8.69 & 11.24 & 20.63 & $9.58 *$ & 12.47 & $*$ \\
\hline
\end{tabular}

Note: \& indicates BMD and $\mathrm{BMD}_{\mathrm{U}}$ for neuroblastoma could not be determined for $40 \%$ of simulations; *indicates lower confidence bound on BMD is obtained with a BMDS module that is still under development. This software does not calculate $\mathrm{BMD}_{\mathrm{U}}$.

(Figure 5.1) is also bounded away from 0); consequently the distribution of BMD and extra risk for combined tumors is well defined.

Tables 5.2 and 5.3 show individual and combined BMDs and extra risks calculated by two approaches: WinBUGS and BMDS (US EPA 2006). Lower confidence bound on combined BMD (Table 5.2) is obtained by an experimental module of BMDS, which is still undergoing testing. That module uses a profile likelihood approach similar to the current approach for an individual 
TABLE 5.3 Statistics for individual and combined extra risks for the nectorine example

\begin{tabular}{|c|c|c|c|c|c|c|}
\hline \multirow[b]{2}{*}{ Approach } & \multicolumn{3}{|c|}{ WinBUGS } & \multicolumn{3}{|c|}{ BMDS } \\
\hline & $\begin{array}{c}5 \text { th } \\
\text { Percentile }\end{array}$ & Mean & $\begin{array}{c}\text { 95th } \\
\text { Percentile }\end{array}$ & $\begin{array}{c}5 \text { th } \\
\text { Percentile }\end{array}$ & MLE & $\begin{array}{c}95 \text { th } \\
\text { Percentile }\end{array}$ \\
\hline Adenoma & 0.055 & 0.076 & 0.099 & 0.048 & 0.072 & 0.097 \\
\hline Neuroblastoma & 0 & 0.011 & 0.028 & 0.007 & 0.016 & 0.028 \\
\hline Combined & 0.059 & 0.086 & 0.135 & $*$ & 0.088 & $0.115^{*}$ \\
\hline
\end{tabular}

Note: For *, see caption for Table 5.2.

tumor in BMDS. Also note that for the extra risk WinBUGS provides estimates of average extra risk, whereas BMDS provides estimates of MLE extra risk.

Interestingly, while $\mathrm{BMD}_{\mathrm{L}} \mathrm{S}$ calculated by two methods are quite close for individual tumors, there is a difference in combined $\mathrm{BMD}_{\mathrm{L}}$ (Table 5.2) and upper bound on extra risk (Table 5.3).

It is clear from the figures that risk of adenoma dominates the risk of neuroblastoma. However, even for this example, with markedly unequal risks from the tumors, the extra risk can be substantially greater when risks are combined. For example, Table 5.3 shows that the 95 th percentile risk at $11 \mathrm{ppm}$ is about $35 \%$ greater for combined risk than for adenoma alone (WinBUGS computation). The difference for average risk is less pronounced (13\% larger) but still nontrivial.

\section{DISCUSSION}

In this chapter, an application of Bayesian methods for calculating probability distributions for composite cancer risk estimates is proposed. Advantages of the proposed approach are that the concept can easily be extended to a more general case, such as a Bayesian hierarchical model with covariates, and computations are easy to implement.

As NRC (1994) stated, ignoring issues about combining tumors could lead to underestimation of risk. In this example with an order of magnitude difference between individual tumors, the underestimation was moderate, compared to other uncertainties inherent to extrapolating risk from animals to humans. It is easy to see that in some situations (e.g., more than two tumors or tumors of similar potency), ignoring additional tumors could lead to a serious underestimation of risk from a toxic substance.

It is very important to choose an appropriate prior when parameter estimates are either on the boundary of the parameter space or are near the boundary for a finite sample. We chose a prior that is a mixture of a diffuse 
continuous prior over the nonnegative real line and a small point mass at 0 . In limited Monte Carlo simulations such mixture priors performed reasonably well in a frequentist sense. Using a continuous prior over the nonnegative real line could lead to undesirable frequentist properties of the posterior when model parameters are at or near the boundaries of parameter space (zero for parameters of the multistage model). However, further research is needed to determine the optimal way of allocating prior density to the point mass at 0 and the continuous nonnegative part.

The approach presented in this chapter provides statistical uncertainty in risk estimates conditional on the data set and multistage models of specified order. The approach can be straightforwardly extended to models other than the multistage model; Bayesian model selection could also be used. However, the uncertainty discussed in this article is only for a particular dose-response model and does not address uncertainty associated with the selection of a particular model, or a set of models.

The approach assumes independence of tumors given dose. Independence may not be true for some types of tumors. Possible next steps include investigation of consequences of violation of independence assumption, and ways to evaluate and incorporate dependence in the methodology.

\section{REFERENCES}

Bogen KT. 1990. Uncertainty in Environmental Health Risk Assessment. London: Taylor and Francis.

Brooks S. 1998. Markov chain Monte Carlo method and its application. Statistician 47(1):69-100.

Casella G, George E. 1992. Explaining the Gibbs sampler. Am Statistician 46(3): 167-74.

Chib S, Greenberg E. 1995. Understanding the Metropolis-Hastings algorithm. Am Statistician 49(4):327-35.

Crump KS. 1984. A new method for determining allowable daily intakes. Fund Appli Toxicol 4:845-71.

Gelfand AE, Smith AFM, Lee T-M. 1992. Bayesian analysis of constrained parameter and truncated data problems using Gibbs sampling. J Am Statist Assoc 418: 523-32.

Gelman A, Carlin JB, Stern HS, Rubin DB. 2004. Bayesian Data Analysis, 2nd ed. London: Chapman and Hall.

Gilks W, Richardson S, Spiegelhalter D. 1998. Markov Chain Monte Carlo in Practice. New York: Chapman and Hall/CRC.

Kopylev L, Chen C, White P, 2007. Towards quantitative uncertainty assessment for cancer risks: central estimates and probability distributions of risk in dose-response modeling. Regul Toxicol Pharmacol 49:203-7.

NRC (National Research Council). 1994. Science and Judgement in Risk Assessment. Washington, DC: National Academy Press. 
Smith A, Gelfand A. 1992. Bayesian statistics without tears: a sampling-resampling perspective. Am Statistician 46(2):84-89.

Spiegelhalter D, Thomas A, Best N. 2003. WinBUGS Version 1.4 User Manual. http:// www.mrc-bsu.cam.ac.uk/bugs/winbugs/manual14.pdf.

US Environmental Protection Agency. 2005. Guidelines for Carcinogen Risk Assessment. EPA/630/P-03/001F.

US Environmental Protection Agency. 2006. Help Manual for Benchmark Dose Software-Version 1.4. EPA 600/R-00/014F. 